\title{
Efficacy of Different Endodontic Irrigant Activation Techniques on Debris Removal from the Mesial Root Canal System of Mandibular Molars
}

\author{
Sara Alsubait ${ }^{1} \odot$, Yara Al-Shibani ${ }^{2}$, Nouf Al-Shehri ${ }^{3}$, Norah AlNowaiser ${ }^{4}$, Tagreed Alojaymi ${ }^{5}$, Nassr Almuflehi ${ }^{6}$, Mazen Alkahtany ${ }^{7}$
}

\begin{abstract}
Aim and objective: This study was conducted to compare debris removal from the mesial canal system with four different irrigation techniques: sonic activation with EDDY, passive ultrasonic irrigation (PUI), mechanical activation with the XP-endo Finisher (XPF), and manual dynamic irrigation (MDI) with gutta-percha.

Materials and methods: Fifty-six extracted mandibular molars with isthmus in the apical $5 \mathrm{~mm}$ in mesial roots were sectioned horizontally at $3 \mathrm{~mm}$ and $5 \mathrm{~mm}$ from the apex. The sections were reassembled, and the mesial canals were prepared chemomecahanically. Specimens were allocated randomly into four groups according to the final irrigation technique. Standardized images of the coronal aspect of cross sections were obtained using a digital stereomicroscope before and after final irrigation protocols to calculate the percentage of debris elimination from the canal system after final irrigation.

Results: The use of EDDY, PUI, and XPF exhibited significant reductions in debris compared with MDI at $5 \mathrm{~mm}(p<0.00)$ and at $3 \mathrm{~mm}(p<0.00)$. Furthermore, no significant difference was noted among EDDY, PUI, and XPF. For intragroup analysis, no statistically significant difference in the percentage of debris elimination was noted between $3 \mathrm{~mm}$ and $5 \mathrm{~mm}$ in all four groups.

Conclusion: All groups showed a reduction in debris after the final irrigation protocol. However, the use of EDDY, PUI, and XPF after cleaning and shaping yielded a significant reduction in debris compared with MDI.

Clinical significance: The use of PUI, XPF, and EDDY as an adjunctive irrigation step presented similar results in improving canal cleanliness, which is hypothesized to affect the treatment outcome.

Keywords: Debris removal, Isthmus, Root canal irrigation, Sonically activated irrigation, Ultrasonically activated irrigation, XP-endo Finisher. The Journal of Contemporary Dental Practice (2021): 10.5005/jp-journals-10024-3054
\end{abstract}

\section{INTRODUCTION}

Complete debridement of the root canal system is a challenge due to the presence of a complex canal anatomy. ${ }^{1}$ Most of the available systems for canal mechanical preparation result in $16-56 \%$ of the walls untouched. ${ }^{2,3}$ Uninstrumented areas may harbor pulp tissue, bacteria and their byproducts, and dentinal debris that might lead to treatment failure. ${ }^{4}$ Therefore, irrigation with the disinfecting solution is mandatory to improve disinfection during canal preparation. ${ }^{5}$

Manual irrigation with a syringe remains the most commonly used irrigation method. ${ }^{6}$ However, it has been shown that syringe irrigation is unable to remove debris from apical irregularities.? Therefore, different techniques and devices were developed to activate the irrigant mainly after the completion of root canal preparation to enhance the canal debridement. A simple method for irrigant activation is moving a well-fitted gutta-percha cone in short, gentle strokes to agitate the irrigant in a technique known as manual dynamic irrigation (MDI). This technique is effective in debris removal. ${ }^{8}$ Passive ultrasonic irrigation (PUI) is an ultrasonic activation of the irrigant with a small, oscillating instrument placed in the root canal. ${ }^{9}$ PUI can induce acoustic streaming and cavitation. ${ }^{9,10}$ Previous studies showed that PUI promoted better cleaning of the canal compared with conventional irrigation. ${ }^{11,12}$ XPF (FKG Dentaire, La Chaux-de-Fonds, Switzerland) is a size 25 nontapered instrument that was introduced to improve endodontic disinfection. When rotated and moved up and down in the canal,

\footnotetext{
1,7Department of Restorative Dental Science, College of Dentistry, King Saud University, Riyadh, Kingdom of Saudi Arabia

${ }^{2-5}$ Department of Restorative Dental Science, College of Dentistry, King Saud University, Riyadh, Kingdom of Saudi Arabia

${ }^{6}$ Department of Preventive Dental Sciences, College of Dentistry, King Saud University, Riyadh, Kingdom of Saudi Arabia
}

Corresponding Author: Sara Alsubait, Department of Restorative Dental Science, College of Dentistry, King Saud University, Riyadh, Kingdom of Saudi Arabia, e-mail address: salsubait@ksu.edu.sa

How to cite this article: Alsubait S, Al-Shibani Y, Al-Shehri N, et al. Efficacy of Different Endodontic Irrigant Activation Techniques on Debris Removal from the Mesial Root Canal System of Mandibular Molars. J Contemp Dent Pract 2021;22(3):231-236.

Source of support: Nil

Conflict of interest: None

XPF might be able to clean the untouched areas. Previous studies reported that both PUI and XPF have similar effectiveness in the removal of hard tissue debris. ${ }^{11,13}$

Recently, EDDY (EDDY, VDW, Munich, Germany), a new sonic device, was launched. EDDY is a flexible, smooth polyamide tip with a size of 25 and 0.04 taper. Typically, sonic devices operate at $1-8 \mathrm{kHz} .{ }^{14}$ EDDY is employed with sonic activation at a frequency of $6000 \mathrm{~Hz}$ using a conventional air scaler. The manufacturer claims that the high-frequency vibration induces acoustic streaming and 
cavitation, and these two effects are generated by PUI and are attributed to its enhancement in cleaning efficiency.

Several earlier studies have evaluated the effectiveness of EDDY on the removal of debris from the root canal system. ${ }^{15-19}$ However, the evaluation of its efficacy on teeth with complex canal anatomy has been reported in few studies with conflicting results, ${ }^{15,16,19}$ which highlights the need for further research. Thus, the aim of the present study was to compare the efficacy of four different irrigation techniques in vitro: sonic activation with EDDY, $\mathrm{PUI}$, mechanical activation with the XPF, and MDI with gutta-percha on debris removal from a mesial canal system. The null hypothesis tested was that there is no difference among the four irrigation activation techniques on debris removal from the mesial root canals of mandibular molars.

\section{Materials and Methods Ethical Approval}

The study methodology was approved by the Institutional Review Board (E-20-4663) and the College of Dentistry Research Center of King Saud University (FR 0549), Riyadh, Kingdom of Saudi Arabia.

\section{Sample Size Estimation and Teeth Selection}

The sample size was calculated using $G^{*}$ Power 3.1.9.4 software at an alpha error probability of 0.05 with an effect size of 0.45 and power of $82 \%$. Power analysis showed that the required sample size per group was 14 teeth.

Human mandibular molars that had been extracted for reasons unrelated to the current study were selected. The inclusion criteria were teeth with fully formed apices, moderately curved canals $\left(10^{\circ}-\right.$ $25^{\circ}{ }_{,}{ }^{20}$ teeth without cracks, teeth without calcifications, and teeth with no previous endodontic treatment as confirmed by digital radiographs. Selected teeth were scanned in a microcomputed tomography $(\mu-\mathrm{CT})$ device (1173 Micro-CT, Bruker SkyScan, Kontich, Belgium) at $85 \mathrm{kV}, 90 \mu \mathrm{A}, 16.98 \mu \mathrm{m}$ pixel size, 0.25 brass filter, and $360^{\circ}$ rotation around the vertical axis with a rotation step of $0.4^{\circ}$ to confirm the presence of an isthmus in the apical $5 \mathrm{~mm}$ in mesial roots. Teeth that met the criteria were randomly distributed into four groups using a computer algorithm (www.random.org) according to the final irrigation protocol. The Kolmogorov-Smirnov test showed a normal distribution of the data, and Levene's test confirmed the intergroup homogeneity regarding the volume of the canals and isthmus areas in the apical $5 \mathrm{~mm}$ in mesial roots $(p>0.05)$. Each tooth was coded and stored in saline solution.

\section{Specimen Preparation}

The pulp chambers were accessed conventionally. The occlusal cusps were flattened to obtain a stable reference point during chemomechanical preparation. The coronal one-third of the mesial canals was flared using a ProTaper Gold Sx file (Dentsply Maillefer, Ballaigues, Switzerland). Working length (WL) was recorded at a distance $0.5 \mathrm{~mm}$ shorter from the length at which a size $10 \mathrm{~K}$-file (Dentsply Maillefer, Ballaigues, Switzerland) was visible at the mesiobuccal and mesiolingual canal terminus. Afterward, flowable composite resin (SDI wave, Bayswater, Australia) was used to seal the apices of the mesial roots and distal canal orifice. Canals were irrigated with saline followed by the placement of cotton pellets and temporary filling material (Coltosol F, Coltène, Altstätten, Switzerland) to seal the access opening.

The experimental model described by Passalidou et al. ${ }^{12}$ was used in the present study. Before mounting, the apical $3 \mathrm{~mm}$ of the roots were immersed in methylene blue for the identification of the apex during sectioning. Each tooth specimen was embedded vertically in a rubber mold (with $30 \mathrm{~mm}$ edges) containing acrylic resin (Vertex Orthoplast; Vertex-Dental, Zeist, The Netherlands) $2 \mathrm{~mm}$ apical to the occlusal surface. After the resin had hardened, four holes were drilled at the corners of the blocks, and then each block was sectioned axially at 3 and $5 \mathrm{~mm}$ from the apex (Isomet, Buehler Ltd., Lake Blu, New York, United States). Standardized images of the coronal aspect of each section were obtained using a stereomicroscope digital microscope (KH-7700, Hirox, Japan) at $50 \times$ magnification. These images comprised the prepreparation images. The specimens were reassembled and firmly tightened with the help of guide pins and metal bolts in the predrilled shafts.

\section{Canal Preparation}

After the removal of the temporary filling and cotton pellet, a size $10 \mathrm{~K}$-file was placed inside the mesiobuccal and mesiolingual canals to verify WL and proper assembly. ProTaper Gold instruments were used according to the manufacturer's instructions up to size 30 with a 0.09 taper (F3). Each instrument was discarded after instrumenting two teeth or if an unwinding occurred. After each instrument, $2 \mathrm{~mL}$ of $2.5 \%$ sodium hypochlorite $(\mathrm{NaOCl})$ was applied using a 30-gauge tip needle (Ultradent Inc., South Jordan, Utah, United States) inserted as deep as possible without binding, respecting the maximum distance of $1 \mathrm{~mm}$ from the WL. After completion of instrumentation, each canal was irrigated with $2 \mathrm{~mL}$ of $\mathrm{NaOCl}$ with the needle placed $1 \mathrm{~mm}$ short of the WL and dried with paper points (Dentsply Maillefer). The access opening was sealed. The specimen was disassembled, and images of the coronal surface of each section were obtained as previously described (postpreparation images).

\section{Final Irrigation Protocol}

The specimen sections were reassembled, and all metal bolts were firmly tightened. A size $10 \mathrm{~K}$-file was placed inside the mesial canals to verify proper assembly. Two milliliters of $\mathrm{NaOCl}$ was applied to the root canals and pulp chamber with a syringe. Afterward, the irrigant was activated according to the assigned supplementary irrigation protocols as follows:

- Sonic activation with EDDY (EDDY): The irrigant was activated with an EDDY tip placed $1 \mathrm{~mm}$ short of the $\mathrm{WL}$ and operated by an air scaler handpiece (SonicFlex, KaVo, Biberach, Germany) at a frequency of $6000 \mathrm{~Hz}$. The EDDY tip was moved vertically (2-4 mm) inside the canal. Each EDDY tip was used in two teeth and then discarded.

- Passive ultrasonic irrigation (PUI): A noncutting, 20/0.00, ultrasonic stainless steel tip (Irrisafe; Satelec Acteon Group, Merignac, France) mounted on an ultrasonic device (P5 Newtron; Satelec) at a power setting of 8 was placed $1 \mathrm{~mm}$ short of the WL and moved up and down ( $2-4 \mathrm{~mm}$ ) in vertical motions. Each ultrasonic stainless steel tip was used in two teeth and then discarded.

- XP-endo Finisher (XPF): The XPF instrument was inserted inside the canal and then activated at 800 rpm using slow and gentle 7 to $8 \mathrm{~mm}$ vertical movements up to the WL inside a cabinet with a temperature kept at $37^{\circ} \mathrm{C}$ (Gourmia; Brooklyn, New York, United States). ${ }^{21}$ Each XPF instrument was used in two teeth and then discarded.

- Manual dynamic irrigation (MDI): A ProTaper F3 gutta-percha cone (Dentsply Maillefer) was inserted into the WL. In and out movements were performed manually. 
Irrigant in all groups was activated for 30 seconds, and then the canal was irrigated with an additional $2 \mathrm{~mL} \mathrm{NaOCl}$ followed by activation of the irrigant for an additional 30 seconds. All groups received a final irrigation with $2 \mathrm{~mL} \mathrm{NaOCl}$ in each canal using a 30-gauge needle placed $1 \mathrm{~mm}$ short of $\mathrm{WL}$, and canals were then dried with paper points. Afterward, each specimen was disassembled, and images of each section were obtained as described above. These images comprised the postirrigant activation images. A single operator performed all procedures.

\section{Method of Evaluation}

The full color images were viewed, and the area of debris was marked and quantified by one blinded operator who was not involved in canal preparation using ImageJ software (National Institutes of Health, Bethesda, Maryland, United States). Debris was defined as any material present on the canal walls, in the canal lumen, or in the isthmus. For each section, the following formula was used to calculate the amount of debris removed after the use of the final irrigation protocol: $\left[\left(D_{\mathrm{BIA}}-D_{\mathrm{AIA}}\right) / D_{\mathrm{BIA}}\right]^{*} 100$, where $D_{\mathrm{BIA}}$ is the amount of debris before irrigant activation, and $D_{\mathrm{AIA}}$ is the amount of debris after irrigant activation.

\section{Statistical Analysis}

The normality of the data was confirmed by the Shapiro-Wilk test. Analysis of variance was performed and complemented by Tukey's test for pairwise comparisons to compare the means of the percentage of removed debris between groups. The $t$-test was used for intragroup analysis to compare the percentage reduction in debris at 3 and $5 \mathrm{~mm}$. The significance level was set at $p<0.05$. Data were statistically analyzed using SPSS software (version 21.0, SPSS IBM, Armonk, New York, United States).

\section{ResULts}

Figures 1 and 2 show representative images of the canal system after sectioning, cleaning, and shaping, and after final irrigation at $5 \mathrm{~mm}$ and $3 \mathrm{~mm}$ from the WL, respectively. The mean and standard deviation of the percentage of eliminated debris for all groups are shown in Table 1.

All groups showed a reduction in debris after the final irrigation protocol. However, the use of EDDY, PUI, and XPF after cleaning and shaping yielded a significant reduction in debris compared with $\mathrm{MDI}$ at $5 \mathrm{~mm}(p<0.00)$ and at $3 \mathrm{~mm}(p<0.00)$. Furthermore, no significant difference was noted among EDDY, PUI, and XPF at
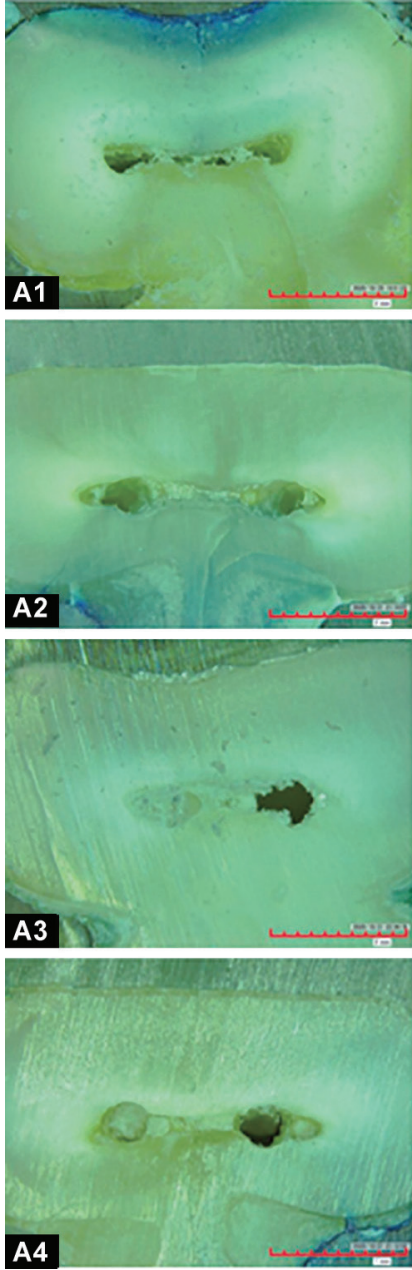
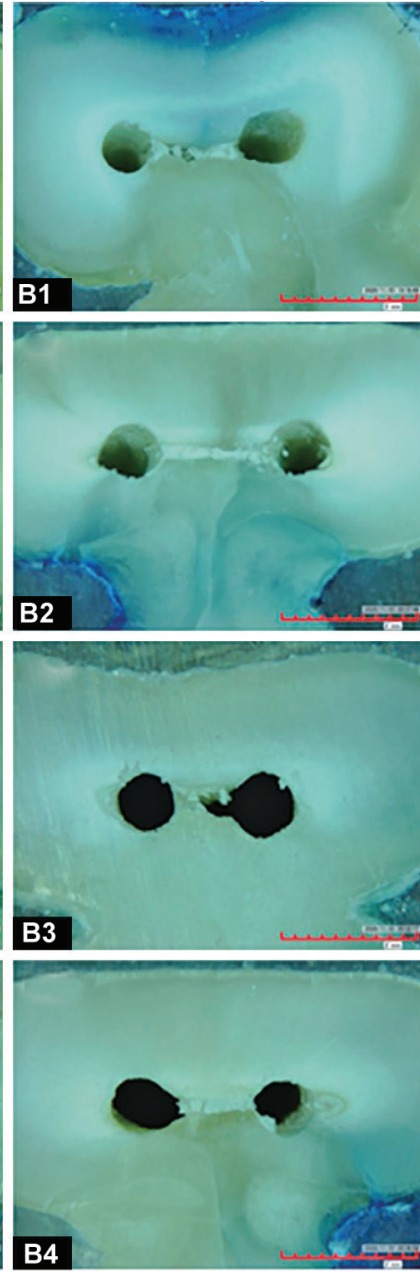
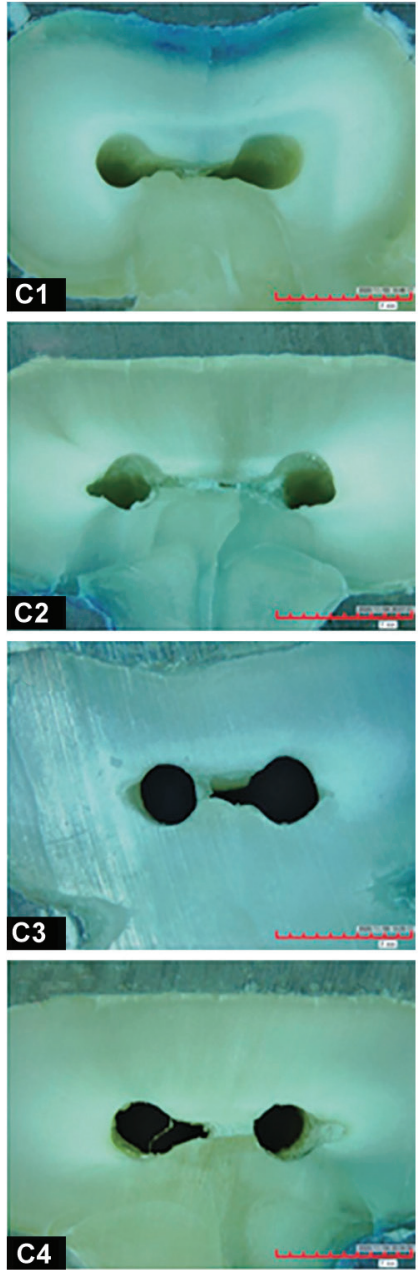

Figs $1 \mathrm{~A}$ to $\mathrm{C}$ : Representative images of root cross sections at $5 \mathrm{~mm}(\mathrm{~A})$ before canal preparation, (B) after canal preparation, and (C) after supplementary irrigant activation with a (1) EDDY, (2) passive ultrasonic, (3) XPF, or (4) manual dynamic activation with gutta-percha cone 

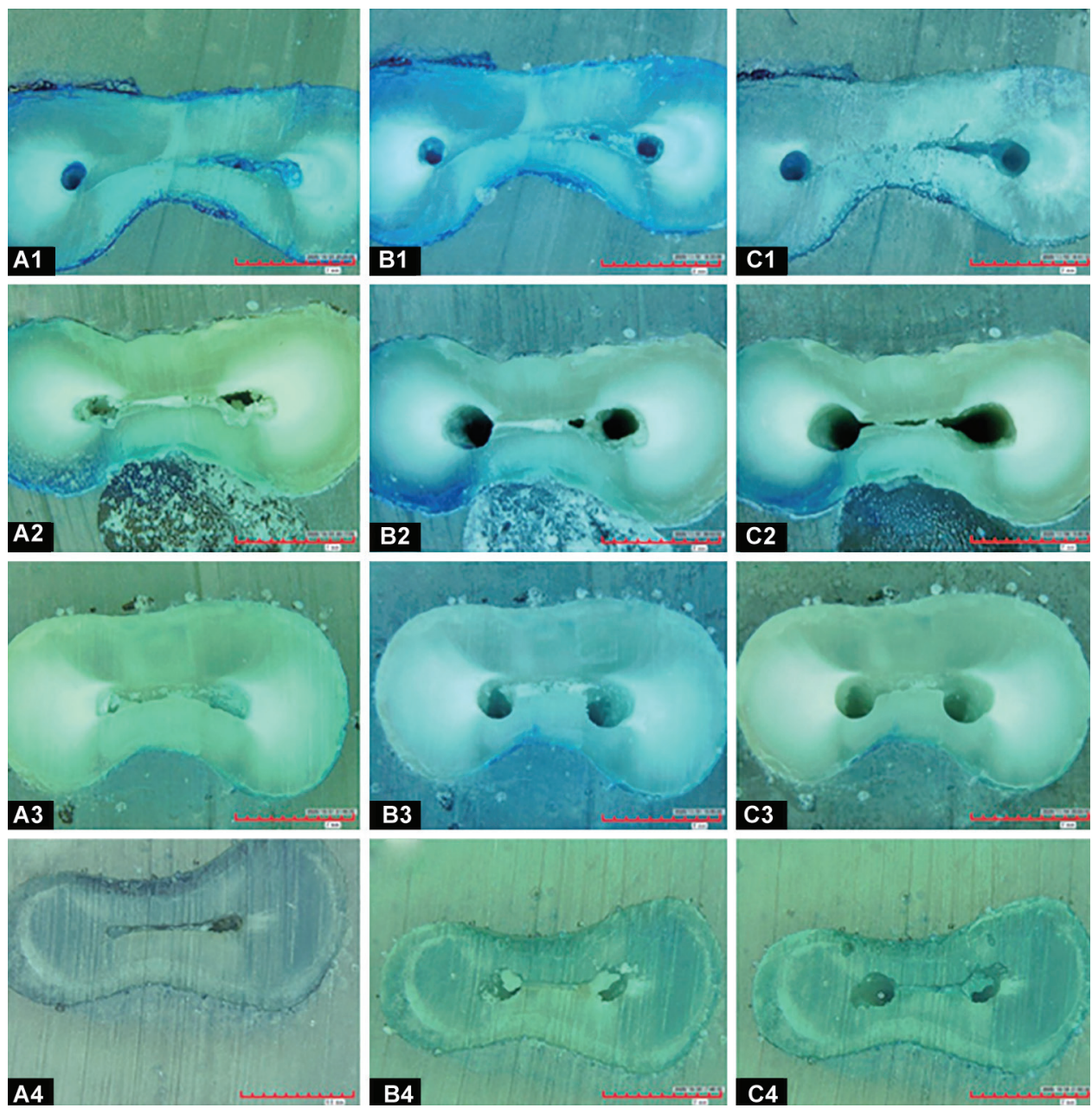

Figs 2A to C: Representative images of root cross sections at $3 \mathrm{~mm}(\mathrm{~A})$ before canal preparation, (B) after canal preparation, and (C) after supplementary irrigant activation with a (1) EDDY, (2) passive ultrasonic, (3) XPF, or (4) manual dynamic activation with gutta-percha cone

Table 1: Means and standard deviations for the percentage of debris reduction after the use of different final irrigation protocols at $3 \mathrm{~mm}$ and $5 \mathrm{~mm}$ from $\mathrm{WL}$

\begin{tabular}{lllll}
\hline & \multicolumn{4}{c}{ Final irrigation protocols } \\
\cline { 2 - 5 } & $E D D Y$ & $P U I$ & $X P F$ & $M D I$ \\
\hline $3 \mathrm{~mm}$ & $86.42 \pm 2.75^{\mathrm{a}}$ & $87.33 \pm 3.99^{\mathrm{a}}$ & $87.32 \pm 3.84^{\mathrm{a}}$ & $45.82 \pm 3.97^{\mathrm{b}}$ \\
$5 \mathrm{~mm}$ & $88.88 \pm 4.43^{\mathrm{a}}$ & $88.39 \pm 4.3^{\mathrm{a}}$ & $87.85 \pm 5.98^{\mathrm{a}}$ & $49.12 \pm 4.65^{\mathrm{b}}$ \\
" $p$ "value & 0.09 & 0.51 & 0.78 & 0.00 \\
\hline
\end{tabular}

Different superscripted lowercase letters indicate statistically significant differences in the percentage of debris reduction between EDDY, PUI, XPF, and MDI within each section level $(p<0.05)$; PUI, passive ultrasonic irrigation; XPF, XP-endo Finisher; MDI, manual dynamic irrigation

$5 \mathrm{~mm}$ (EDDY vs PUI: $p=0.91$, EDDY vs XPF: $p=0.92$, and PUI vs XPF: $p=1$ ) or at $3 \mathrm{~mm}$ (EDDY vs PUI: $p=0.99$, EDDY vs XPF: $p=0.94$, and PUI vs XPF: $p=0.99$ ). For intragroup analysis, no statistically significant difference in the percentage of debris elimination was noted between 3 and $5 \mathrm{~mm}$ in all four groups (Table 1).

\section{Discussion}

Complete disinfection of mandibular molars is difficult due to the complex canal anatomy. One of the major complexities in molars is the presence of isthmuses. The isthmus is defined as a narrow connection between two or more canals in the same root. ${ }^{22}$ It has been reported that isthmuses are found in 87 and $66 \%$ of the mandibular first and second molars, respectively. ${ }^{23}$ The highest incidence of isthmuses was observed at 3 to $6 \mathrm{~mm}$ from the apex. ${ }^{24}$ In clinical practice, the isthmus area is difficult to access and debride using mechanical instrumentation. Therefore, isthmus-containing mesial roots of mandibular molars were selected for this study. To reduce the potential anatomical biases between the groups, teeth were scanned before canal preparation, and the volume of the canals and isthmus areas in the apical $5 \mathrm{~mm}$ were statistically analyzed. The irrigant penetration into the apical one-third of the canal is related to the apical preparation size. Size 30 is the minimum instrumentation size that is needed to achieve proper irrigant 
penetration into the apical third..$^{25}$ Therefore, in the present study, preparation of the F3 file was performed.

Different methods have been used to assess the cleanliness of root canals after the use of different irrigant activation techniques. Micro-CT has been used in previous studies. ${ }^{11,13,16}$ However, the major limitation of this technology when used for calculating the amount of debris is that the organic debris present in the root canal system cannot be viewed on radiographic images. ${ }^{26}$ The use of root sectioning and microscopic evaluation methodology was used in other studies to overcome this limitation. ${ }^{12,27,28}$ Thus, this methodology was selected for the present study. The K-Kube model that was described by Passalidou et al. ${ }^{12}$ was used in this study. In addition to the ability to evaluate soft and hard tissue debris, it allows each tooth to serve as its own control by evaluating the same section at each treatment procedure. Furthermore, the K-Kube model provides a closed system that more accurately simulates in vivo conditions.

In the present study, none of the tested supplementary irrigant activation techniques was able to remove debris completely from the root canals. The finding is consistent with previous studies. ${ }^{12,15,16}$ However, the use of EDDY, PUI, and XPF resulted in significantly higher percentages of debris reductions compared with MDI. Hence, the null hypothesis was rejected. MDI has the advantage of being a simple and low-cost technique. However, MDI was the least effective in debris removal from the canal system, which might be explained by the repeated friction of the debris against the canal walls with a well-fitted gutta-percha that produces more particular agglomeration on canal walls. ${ }^{29}$ This observation was consistent with previous studies regarding debris and smear layer removal. . $^{12,30}$

PUI has been reported to reduce debris in the root canal after canal preparation, ${ }^{11,12}$ which is consistent with the results of the present study. The efficacy of PUI is related to the high-frequency vibration that induces acoustic streaming and cavitation. In the current study, XPF presented similar effectiveness to PUI in the reduction of hard and soft debris. Leoni et al. ${ }^{11}$ and De-Deus et al. ${ }^{13}$ evaluated the removal of hard tissue debris using micro-CT technology and reported similar findings. XPF is an instrument that is made of a highly flexible proprietary alloy. XPF has a small core size and a zero taper. Most likely, when activated inside the canal, the helical movement of the file may touch and displace the debris inside the canal system. ${ }^{11}$

Conflicting results have been reported in the literature regarding the efficacy of sonic activation techniques in removing debris from the root canal system. ${ }^{14,31}$ Compared with ultrasonic activation, sonic irrigation devices operate at lower frequencies. EDDY is a newly introduced ultrasonic activation device that operates at high frequency $(6000 \mathrm{~Hz})$. In the present study, the cleaning ability of EDDY is similar to that of PUI. This finding is consistent with those reported by Haupt et al. ${ }^{15}$ This finding might be explained by the high frequencies in EDDY compared with other sonic irrigation devices, which is expected to improve the irrigant flow to displace the debris. However, this finding is in disagreement with Linden et al., ${ }^{16}$ where PUI resulted in a significantly higher percentage of reduction compared to EDDY. Hard tissue debris removal was evaluated in the Linden et al. ${ }^{16}$ study, whereas hard and soft tissue debris were considered in the present study. To the best of our knowledge, this is the first study that evaluated canal cleanliness after the use of EDDY and XPF supplementary techniques. Both activation techniques showed similar effectiveness in the removal of root canal debris. In the current investigation, no differences in debris reduction were noted between 3 and $5 \mathrm{~mm}$ in all groups.
This finding might be attributed to the final canal preparation size (30.09), which allowed the activation instrument to oscillate freely, followed by the agitation of the irrigant and disruption of the debris.

A major limitation of this in vitro study is that it cannot duplicate the in vivo conditions. However, this study can provide information that can help clinicians select supplementary irrigant activation techniques. From a clinical point of view, the results of the present study can be translated into the fact that the use of PUI, XPF, and EDDY as an adjunctive irrigation step had similar results in improving canal cleanliness, which is hypothesized to affect the treatment outcome. However, the EDDY tip may have an advantage compared to PUI and XPF. The soft polymer tips in EDDY sonic devices would reduce the risk of unintentional dentin removal from the prepared canal system, which can be caused by the use of PUI ${ }^{32}$ and $X P F{ }^{33}$ Further studies are required to assess dentin removal after using sonic activation at high frequency.

\section{Conclusion}

In conclusion, sonic activation with EDDY, PUI, mechanical activation with $X P F$, and $M D I$ with gutta-percha showed a reduction in debris after the final irrigation protocol. However, the use of EDDY, PUI, and XPF after cleaning and shaping yielded significant reductions in debris compared with MDI.

\section{ACKNOWLEDGMENT}

The authors would like to thank the College of Dentistry Research Center at King Saud University for its support in the present project.

\section{OrCID}

Sara Alsubait (1) https://orcid.org/0000-0002-8001-620X

\section{References}

1. Vertucci FJ. Root canal anatomy of the human permanent teeth. Oral Surg Oral Med Oral Pathol 1984;58(5):589-599. DOI: 10.1016/00304220(84)90085-9.

2. Zuolo ML, Zaia AA, Belladonna FG, et al. Micro-CT assessment of the shaping ability of four root canal instrumentation systems in oval-shaped canals. Int Endod J 2018;51(5):564-571. DOI: 10.1111/ iej.12810.

3. Zhao D, Shen Y, Peng B, et al. Root canal preparation of mandibular molars with 3 nickel-titanium rotary instruments: a micro-computed tomographic study. J Endod 2014;40(11):1860-1864. DOI: 10.1016/j. joen.2014.06.023.

4. Ricucci D, Siqueira JF, Bate $A L$, et al. Histologic investigation of root canal-treated teeth with apical periodontitis: a retrospective study from twenty-four patients. J Endod 2009;35(4):493-502. DOI: 10.1016/j.joen.2008.12.014.

5. Haapasalo M, Shen Y, Wang Z, et al. Irrigation in endodontics. Br Dent J 2014;216:299-303. DOI: 10.1038/sj.bdj.2014.204.

6. Dutner J, Mines P, Anderson A. Irrigation trends among American association of endodontists members: a web-based survey. J Endod 2012;38(1):37-40. DOI: 10.1016/j.joen.2011.08.013.

7. Jiang LM, LakB, Eijsvogels LM, et al. Comparison of the cleaning efficacy of different final irrigation techniques. J Endod 2012;38(6):838-841. DOI: 10.1016/j.joen.2012.03.002.

8. Huang TY, Gulabivala K, Ng YL. A bio-molecular film ex-vivo model to evaluate the influence of canal dimensions and irrigation variables on the efficacy of irrigation. Int Endod J 2008;41(1):60-71. DOI: 10.1111/j.1365-2591.2007.01317.x.

9. Ahmad M, Pitt Ford TJ, Crum LA. Ultrasonic debridement of root canals: acoustic streaming and its possible role. J Endod 1987;13(10):490-499. DOI: 10.1016/s0099-2399(87)80016-x. 
10. Roy RA, Ahmad M, Crum LA. Physical mechanisms governing the hydrodynamic response of an oscillating ultrasonic file. Int Endod J 1994;27(4):197-207. DOI: 10.1111/j.1365-2591.1994.tb00254.x.

11. Leoni GB, Versiani MA, Silva-Sousa YT, et al. Ex vivo evaluation of four final irrigation protocols on the removal of hard-tissue debris from the mesial root canal system of mandibular first molars. Int Endod J 2017;50(4):398-406. DOI: 10.1111/iej.12630.

12. Passalidou $S$, Calberson F, De Bruyne $M$, et al. Debris removal from the mesial root canal system of mandibular molars with laseractivated irrigation. J Endod 2018;44(11):1697-1701. DOI: 10.1016/j. joen.2018.06.007.

13. De-Deus G, Belladonna FG, Zuolo ADS, et al. Micro-CT comparison of $\mathrm{XP}$-endo Finisher and passive ultrasonic irrigation as final irrigation protocols on the removal of accumulated hard-tissue debris from oval shaped-canals. Clin Oral Investig 2019;23(7):3087-3093. DOI: 10.1007/s00784-018-2729-y.

14. Sabins RA, Johnson JD, Hellstein JW. A comparison of the cleaning efficacy of short-term sonic and ultrasonic passive irrigation after hand instrumentation in molar root canals. J Endod 2003;29(10):674678. DOI: 10.1097/00004770-200310000-00016.

15. Haupt F, Meinel M, Gunawardana A, et al. Effectiveness of different activated irrigation techniques on debris and smear layer removal from curved root canals: a SEM evaluation. Aust Endod J 2020;46(1):40-46. DOI: 10.1111/aej.12342.

16. Linden $D$, Boone $M$, De Bruyne $M$, et al. Adjunctive steps for the removal of hard tissue debris from the anatomic complexities of the mesial root canal system of mandibular molars: a micro-computed tomographic study. J Endod 2020;46(10):1508-1514. DOI: 10.1016/j. joen.2020.05.009.

17. Plotino G, Grande NM, Mercade M, et al. Efficacy of sonic and ultrasonic irrigation devices in the removal of debris from canal irregularities in artificial root canals. J Appl Oral Sci 2019;27:e20180045. DOI: 10.1590/1678-7757-2018-0045.

18. Urban K, Donnermeyer D, Schafer E, et al. Canal cleanliness using different irrigation activation systems: a SEM evaluation. Clin Oral Investig 2017;21(9):2681-2687. DOI: 10.1007/s00784-017-2070-x.

19. Rodig T, Koberg C, Baxter S, et al. Micro-CT evaluation of sonically and ultrasonically activated irrigation on the removal of hardtissue debris from isthmus-containing mesial root canal systems of mandibular molars. Int Endod J 2019;52(8):1173-1181. DOI: 10.1111/ iej.13100.

20. Schneider SW. A comparison of canal preparations in straight and curved root canals. Oral Surg Oral Med Oral Pathol 1971;32(2):271-275. DOI: 10.1016/0030-4220(71)90230-1.

21. Silva E, Belladonna FG, Zuolo AS, et al. Effectiveness of XP-endo Finisher and $\mathrm{XP}$-endo Finisher $\mathrm{R}$ in removing root filling remnants: a micro-CT study. Int Endod J 2018;51(1):86-91. DOI: 10.1111/iej. 12788.

22. Weller RN, Niemczyk SP, Kim S. Incidence and position of the canal isthmus. Part 1. Mesiobuccal root of the maxillary first molar. J Endod 1995;21(7):380-383. DOI: 10.1016/s0099-2399(06)80975-1.

23. Estrela C, Rabelo LE, de Souza JB, et al. Frequency of root canal isthmi in human permanent teeth determined by cone-beam computed tomography. J Endod 2015;41(9):1535-1539. DOI: 10.1016/j. joen.2015.05.016.

24. Teixeira FB, Sano CL, Gomes BP, et al. A preliminary in vitro study of the incidence and position of the root canal isthmus in maxillary and mandibular first molars. Int Endod J 2003;36(4):276-280. DOI: 10.1046/j.1365-2591.2003.00638.x.

25. Khademi A, Yazdizadeh M, Feizianfard M. Determination of the minimum instrumentation size for penetration of irrigants to the apical third of root canal systems. J Endod 2006;32(5):417-420. DOI: 10.1016/j.joen.2005.11.008.

26. Paque F, Laib A, Gautschi H, Zehnder M. Hard-tissue debris accumulation analysis by high-resolution computed tomography scans. J Endod 2009;35(7):1044-1047. DOI: 10.1016/j. joen.2009.04.026.

27. Duque JA, Duarte MA, Canali LC, et al. Comparative effectiveness of new mechanical irrigant agitating devices for debris removal from the canal and isthmus of mesial roots of mandibular molars. J Endod 2017;43(2):326-331. DOI: 10.1016/j.joen.2016.10.009.

28. Thomas AR, Velmurugan N, Smita S, et al. Comparative evaluation of canal isthmus debridement efficacy of modified EndoVac technique with different irrigation systems. J Endod 2014;40(10):1676-1680. DOI: 10.1016/j.joen.2014.05.014.

29. Shahravan A, Haghdoost AA, Adl A, et al. Effect of smear layer on sealing ability of canal obturation: a systematic review and metaanalysis. J Endod 2007;33(2):96-105. DOI: 10.1016/j.joen.2006.10.007.

30. Ribeiro EM, Silva-Sousa YT, Souza-Gabriel AE, et al. Debris and smear removal in flattened root canals after use of different irrigant agitation protocols. Microsc Res Tech 2012;75(6):781-790. DOI: 10.1002/ jemt.21125.

31. Capar ID, Aydinbelge HA. Effectiveness of various irrigation activation protocols and the self-adjusting file system on smear layer and debris removal. Scanning 2014;36(6):640-647. DOI: 10.1002/sca.21171.

32. Boutsioukis C, Tzimpoulas N. Uncontrolled removal of dentin during in vitro ultrasonic irrigant activation. J Endod 2016;42(2):289-293. DOI: 10.1016/j.joen.2015.09.017.

33. Hristov K, Gateva N, Stanimirov P, et al. Comparative analysis of root dentin loss when using modern mechanical cleaning instruments in immature permanent teeth. Folia Med (Plovdiv) 2020;62(2):352-357. DOI: 10.3897/folmed.62.e39515. 